Ann. Biol. anim. Bioch. Biophys., I968, 8 (3), 449-45I.

\title{
UTILISATION DIGESTIVE DE L'AMIDON DE L'ORGE CHEZ LE RUMINANT
}

\author{
P. THIVEND et M. JOURNET \\ Station de Recherches sur l'Élevage des Ruminants, \\ Centre de Recherches zootechniques et vétérinaires sur les Ruminants, \\ 63 - Theix près Clermont-Ferrand \\ Institut national de la Recherche agronomique
}

La digestion de l'amidon chez le Ruminant donne naissance essentiellement à des acides gras volatils dans le rumen mais aussi à une certaine quantité de glucose dans l'intestin. L'utilisation métabolique de ces nutriments est différente, notamment pour l'engraissement (ARMsTrong et BLAXTER, I96I). Or, dans le cas des régimes riches en aliment concentré, les proportions respectives d'acides gras volatils et de glucose formés semblent varier avec la forme physique, la quantité ingérée et surtout avec la nature de la céréale (KARR, Little et Mitchell, i966; ArMSTrong, Seely et MAC RAE, I967). Nous avons donc entrepris d'étudier l'importance de la digestion dans le rumen et dans l'intestin, des principales céréales utilisées dans l'alimentation du Ruminant. Nous rapportons ici les résultats obtenus avec l'orge.

Nous avons utilisé deux bœufs pesant environ $500 \mathrm{~kg}$, porteurs chacun d'une canule du rumen et d'une canule du duodénum. Nous avons fait varier le rapport foin/concentré de la ration, dans les proportions suivantes : Ioo-o, puis $75-25,40-60,25-75$ et 5-95 sans changer la quantité de matière sèche ingérée qui était égale à celle du foin offert à volonté. La quantité d'amidon ingéré a ainsi varié de 845 à 3 r $5 \circ \mathrm{g}$ par jour. L'aliment concentré $(2 \mathrm{r}, 2 \mathrm{p}$. Ioo de matières azotées totales, 49,6 p. I00 d'amidon) contenait 77,8 p. I00 d'orge, 10,4 p. Ioo de tourteau d'arachide et 10,4 p. Ioo de tourteau de soja. Il était présenté sous forme condensée ; le fourrage était un foin de luzerne (Io,6 p. Ioo de matières azotées totales) sous forme normale. Les animaux ont reçu les cinq régimes au cours de cinq périodes successives comprenant chacune deux semaines d'adaptation et deux semaines de mesure. Nous avons étudié, pour chaque régime, la digestibilité de l'amidon et des autres constituants de la ration, les produits terminaux de la digestion dans le rumen (acides gras volatils, acide lactique) et l'activité amylolytique in vitro du jus de rumen. Nous avons effectué une fois par période et entre deux repas consécutifs, des prélèvements discontinus ( $3 \mathrm{mn}$ toutes les $15 \mathrm{mn}$ ) au niveau du duodénum. A partir de ces prélèvements, nous avons préparé des échantillons représentatifs du débit horaire moyen sur lesquels nous avons dosé l'amidon (Thivend, Mercier et GuILBot, I965).

Le CUD de la ration a augmenté avec le pourcentage d'aliment concentré. Il est passé de 6I,3 à 86,6 pour la matière sèche, de 59,5 à 87,9 pour la matière organique et de 53,2 à 87,3 pour les matières azotées lorsque le rapport foin/concentré variait de 100-0 à 5-95. L'amidon a été entièrement digéré quelle que fût la quantité ingérée. Enfin, la digestibilité de la cellulose brute a peu varié (de 47,3 à 49,5 pour le bouf $n^{\circ} 47^{62}$, de 56,3 à 47 , I pour le bœuf no 57 I2). 


\begin{tabular}{|c|c|c|c|c|c|c|c|c|c|c|c|c|c|}
\hline \multirow{2}{*}{\multicolumn{2}{|c|}{ 蒡苞 }} & \multirow{2}{*}{ 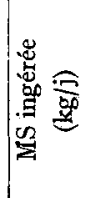 } & \multirow{2}{*}{ 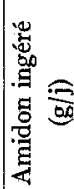 } & \multirow{2}{*}{ ف } & \multicolumn{8}{|c|}{$\begin{array}{l}\text { Acides gras volatils formés } \\
\text { (p. } 100 \text { concentration molaire) }\end{array}$} & \multirow{3}{*}{ 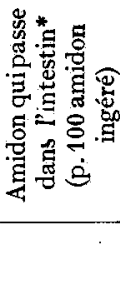 } \\
\hline & & & & & \multicolumn{2}{|c|}{$\mathrm{C}_{2}$} & \multicolumn{2}{|c|}{$\mathrm{C}_{3}$} & \multicolumn{2}{|c|}{$\mathrm{C}_{4}$} & \multicolumn{2}{|c|}{$>C_{2}$} & \\
\hline 100 & 0 & 6,5 & & 1,0 & $70,7^{*}$ & $71,1^{* *}$ & 17,5 & 16,9 & 9,0 & 8,8 & 2,8 & 3,2 & \\
\hline 75 & 25 & 6,3 & 845 & 0,5 & 69,2 & 70,7 & 15,6 & 15,7 & 11,1 & 10,0 & 4,1 & 3,4 & 3,3 \\
\hline 40 & 60 & 7,1 & 1725 & 8,8 & 66,5 & 71,9 & 14,2 & 13,3 & 15,4 & 11,7 & 3,9 & 3,1 & 8,4 \\
\hline 25 & 75 & 6,9 & 2560 & 26,3 & 56,0 & 66,7 & 25,4 & 16,3 & 11,4 & 12,4 & 7,2 & 4,6 & 6,6 \\
\hline 5 & 95 & 7,0 & 3150 & 34,9 & 37,0 & 58,4 & 47,7 & 22,9 & 6,5 & 10,6 & 8,8 & 8,1 & 1,8 \\
\hline & & & & & & & & & & & & & \\
\hline
\end{tabular}

L'orientation des fermentations microbiennes dans le rumen a peu changé tant que la proportion d'aliment concentré ne dépassait pas 60 p. 100. Au-delà de cette valeur, nous avons observé une brusque augmentation de l'activité amylolytique et une modification importante de la composition en acides gras volatils du jus de rumen. L'acidité totale du jus a varié également avec le pourcentage d'aliment concentré ingéré (successivement $9 \mathrm{I}, 4-86,2-106,8-136,1$ mmole par litre). Les quantités d'acide lactique formées ont été faibles ( 7 valeurs supérieures à $0,5 \mathrm{~g} /$ litre sur 72 analyses) pendant toute l'expérience, probablement à cause de l'adaptation progressive des animaux aux différents régimes.

La quantité d'amidon digéré dans l'intestin a varié de $30 \mathrm{~g}$ pour le régime 25-75 à I $70 \mathrm{~g}$ pour le régime 75-25. Cependant, ces valeurs ne représentent qu'un faible pourcentage de l'amidon ingéré (inférieur à 8,5 p. I0o). A partir de 60 p. 100 d'aliment concentré, la part de la digestion intestinale a diminué, ce qui peut s'expliquer par l'augmentation de l'activité amylolytique du jus de rumen. La majeure partie de l'amidon de l'orge est donc transformée en acides gras volatils. ARMSTRonG, SEELy et MAC RAE ( $196_{7}$ ) ont aboutit à la même conclusion avec des moutons recevant des régimes analogues à ceux que nous avons étudiés.

Si l'on veut accroitre la part de la digestion intestinale de l'amidon, par exemple, pour favoriser l'engraissement des bovins et des ovins, il faut remplacer l'orge (broyée et agglomérée), par une autre céréale (maïs) qui échappe en partie aux fermentations du rumen. Au contraire, la digestion quasi complète de l'amidon de l'orge dans le rumen doit être favorable à la production des matières grasses du lait. Cependant, au-delà de 60 p. ıoo d'aliment concentré dans la ration, les déviations des fermentations que nous observons entraînent des baisses du taux butyreux (RÉMOND, données non publiées).

Reçu pour publication en mai 1968.

\section{SUMMARY}

\section{THE UTILIZATION OF BARLEY-STARCH BY RUMINANTS}

Experiments were conducted on two oxen fitted with ruminal and duodenal canulas. 92 to 98 per cent of barley-starch was found to be digested in the rumen, whatever the proportion of the cereal in the diet. The amylolytic activity, the concentration and composition of volatile fatty acids in the rumen were not altered so far as the proportion of high-concentrate rations did not exceed 60 per cent. 


\section{RÉFÉRENCES BIBLIOGRAPHUQUES}

ARMStrong D. G., Blaxter K. L., 196r. The utilization of the energy of carbohydrates by ruminant (in 2 th Symposium on energy metabolism Wageningen, p. 178

Armstrong D. G., Seeley R. C., MaC RaE J. C., I967. Feed carbohydrates. The contribution of the end-products of their digestion to energy supply in the ruminant (IV Symposium internalional sur $l e$ métabolisme énergétique, Varsovie).

KARR M. R., LitTle C. O., Mitchell G. E., Ig66. Starch disappearance from different segments of the digestive tract of steers. J. Anim. Sci., 25, 652-654.

Thivend P., Mercier Ch., Guilbot A., I965. Dosage de l'amidon dans les milieux complexes. Ann. Biol. anim., Bioch., Biophys., 5, 513-526. 